\title{
Erratum: First-Principles Approach to Calculating Energy Level Alignment at Aqueous Semiconductor Interfaces [Phys. Rev. Lett. 113, 176802 (2014)]
}

\author{
Neerav Kharche, James T. Muckerman, and Mark S. Hybertsen \\ (Received 21 April 2017; published 25 May 2017)
}

DOI: 10.1103/PhysRevLett.118.219902

While extending our approach in our Letter to calculate energy level alignment at aqueous $\mathrm{TiO}_{2}$ interfaces, we discovered two problems with our application of the $G W$ methodology. Briefly, in our approach, we used a DFT $+U$ approach for the underlying structure determination and as an input to the $G W$ calculations. Then, $G W$ calculations were carried out at the full-frequency spectrum-self-consistent level. All calculations were performed with the projector augmented wave (PAW) method as it is implemented in VASP. The specific semiconductors considered were GaN and $\mathrm{ZnO}$. The first problem was caused by improper handling of the $U$ parameter when initializing the follow-up $G W$ calculations, leading to a final calculated band gap for $\mathrm{GaN}$ and $\mathrm{ZnO}$ that was too large. Second, we used an older set of pseudopotentials for the PAW method. In a paper that was published while our original manuscript was under review [1], the impact of the completeness of the partial waves representing empty states in the PAW method was analyzed, showing significant issues with the convergence of the $G W$ results for certain materials, particularly ZnO. Specifically, those issues included use of PAW pseudopotentials that deviate too much from norm conservation, an unduly restricted basis set size that constrains the number of empty states available, and use of a small cutoff for the dielectric matrix size that can result in a false convergence with respect to the number of empty states included. Our $G W$ calculations for $\mathrm{GaN}$ and $\mathrm{ZnO}$ were affected by these problems.

We have corrected for the errors in handling the $U$ parameters, affecting $\mathrm{GaN}$ and $\mathrm{ZnO}$ results. We have also completely redone the $G W$ calculations using the new version 5.4 PAW pseudopotentials in VASP, with a much larger basis set and dielectric matrix size (defined by cutoffs of $1000 \mathrm{eV}$ and $800 \mathrm{eV}$, respectively). The underlying DFT $+U$ calculations remain virtually unchanged (levels reported here the same within $0.1 \mathrm{eV}$ ). As before, we extensively sample the calculated $G W$ corrections as a function of the number of empty states but now, with expanded range available and extrapolate to the complete basis set limit. Details appear in the Supplemental Material [2].

The impact of each of these changes is delineated in Table I. Proper handling of the $U$ parameter leads to smaller gaps when combined with $G W$ corrections computated as originally described in our Letter, particularly for ZnO. However, the use of the new PAW pseudopotentials, together with substantially increased cutoffs, increases the band gaps once again and also slightly affects the water results through the basis set change. The net effect is relatively small quantitative corrections to the energy levels previously reported. In particular, the highest occupied states are now systematically deeper, relative to vacuum. The impact on the results previously reported in Figs. 1 and 2 is small, but we show corrected versions below. These changes have no impact on the conclusions in our Letter.

TABLE I. Key energy level characteristics reported previously with corrections due to proper treatment of the $U$ parameter and due to the use of more accurate pseudoptentials in the PAW method together with improved basis sets. For each material, the computed band gap at room temperature is shown, along with the ionization potential (IP) for GaN and $\mathrm{ZnO}$ and the centroid of the highest occupied $E_{1 b 1}$ band for water. Note that the original value of the water band gap $(9.5 \mathrm{eV})$ was estimated from 12 snapshots, while that here is based on 6 snapshots.

\begin{tabular}{lccc}
\hline \hline & & & $\begin{array}{r}\text { New pseudopotentials } \\
\text { and increased basis set }\end{array}$ \\
\hline GaN: Gap & Original & $U$ error corrected & 4.16 \\
IP & 4.16 & 3.86 & 7.22 \\
ZnO: Gap & 6.98 & 6.68 & 3.86 \\
IP & 4.06 & 3.32 & 8.21 \\
Water: Gap & 8.08 & 7.36 & 9.7 \\
$E_{1 b 1}$ & 9.4 & $\ldots$ & -11.66 \\
\hline \hline
\end{tabular}




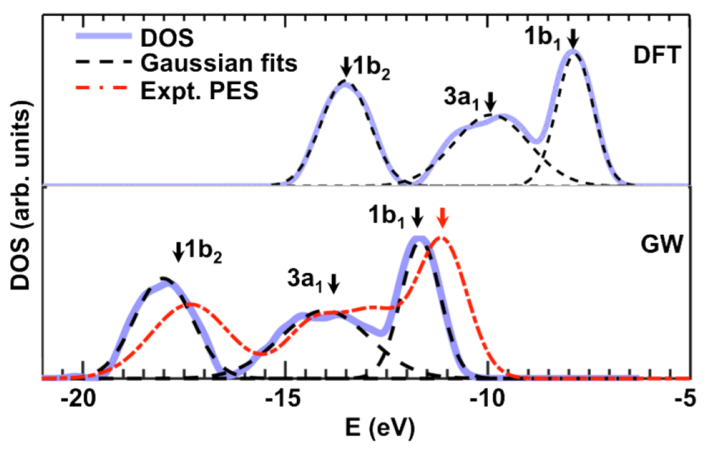

FIG. 1. Replacement for Fig. 3 with corrected results from the $G W$ approach.

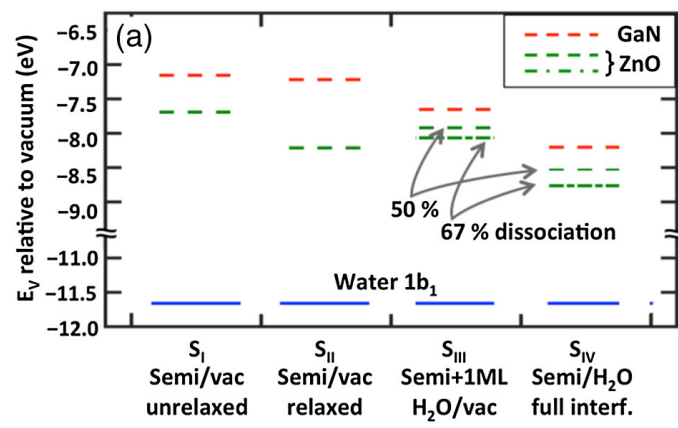

(b)

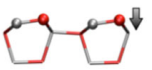

(c)

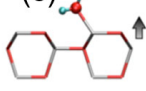

(d)

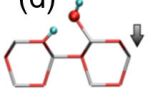

FIG. 2. Replacement for Fig. 4 with corrected results for energy levels.

We thank M. Kaltak and G. Kresse for facilitating our response to the changes to the $G W$ approach described in our Letter and J. L. Lyons for valuable discussions. This work was carried out at Brookhaven National Laboratory under Contract No. DE-SC0012704 with the U.S. Department of Energy, supported by its Office of Basic Energy Sciences (Computational Materials and Chemical Sciences Network program, Division of Chemical Sciences and Scientific User Facilities Division), and utilized resources at the Center for Functional Nanomaterials, Brookhaven National Laboratory, and at the National Energy Research Scientific Computing Center, supported by the Office of Science of the U.S. Department of Energy under Contract No. DE-AC02-05CH11231.

[1] J. Klimes, M. Kaltak, and G. Kresse, Phys. Rev. B 90, 075125 (2014).

[2] See Supplemental Material at http://link.aps.org/supplemental/10.1103/PhysRevLett.118.219902 for details of methods and calculations and plots that illustrate the extrapolation of the $G W$ calculations vs number of empty states. 